\title{
吐温 80-盐-水液一固萃取体系
}

\author{
李步海杨波 \\ (中南民族学院化学系,武汉 430074)
}

\section{关调萃取、吐温 80 、偶氮胂 11 I}

水溶性聚合物聚乙二醇 (PEG)2000 的盐水溶液能分成液一固两相 ${ }^{(2)}$, 可用这一体系进行 金属离子间的萃取分离 ${ }^{[2,3]}$. 我们发现, 聚乙烯吡咯烷酮、吐温类、明胶等聚合物的水溶液在 某些无机盐存在下能分成液一固两相. 水溶性鳌合剂(多用显色剂)及其与金属离子的鳌合物 可在两相间进行分配,借控制溶液酸度等方法,能实现金属离子间的萃取分离, 得到一个新的 萃取体系. 它克服了常用有机溶剂萃取体系的弊端, 固相分离后用水溶解, 可用光度法直接测 定被萃取物浓度. 方法简便、块速. 本文报道吐温 80-盐-水液一固萃取体系的研究结果. 这 一工作尚末见文献报道.

\section{一、实䠯部分}

1. 试剂与仪器不同浓度的吐温（Tween） 80 水溶液。偶氮狷 III 水溶液: $1.29 \times$ $10^{-3} \mathrm{~mol} \cdot \mathrm{L}^{-1} \cdot \mathrm{Zr}(\mathrm{IV}) 、 \mathrm{Sc}(\mathrm{III}) 、 \mathrm{Ni}(\mathrm{II})$ 标准溶液、一氯乙酸、醋酸缓冲溶液均按常规方法配 制.

721 型分光光度计、pHS-2 型酸度计、康氏振荡器.

2. 实验方法于 $25 \mathrm{ml}$ 具塞试管中, 加人 $5.0 \mathrm{ml}$ 一定浓度的吐温 80 水溶液,一定体积 的偶㷋胂 III 水溶液和一定量的金属离子, 高酸度溶液用盐酸调节, $\mathrm{pH} 1.5-6.5$ 溶液借加人一 定体积的缓冲溶液控制酸度, 加人一定量的固体盐, 用水桸释至 $10.0 \mathrm{ml}$. 振荡, 静置几分钟. 水溶液分成液一固两相. 倾出液相, 固相用相同浓度的盐水洗涤, 洗涤液并人液相。固相用水 浴解, 用光度法测定吐温 80 固相或下层盐水相被萃取物浓度, 计算萃取率. 金属离子的光位 测定方法见文献 [4].

\section{二、结果与讨论}

1. 吐温 80 水溶洨的分相条件 图 1 表明, 相同浓度的吐温 80 水溶液,加人不同的盐, 分成液一固两相所需盐的浓度不同. 考虑到 $\mathrm{K}_{2} \mathrm{CO}_{3}$ 在高酸度溶液中会分解及大多金属离子的 碳酸盐不溶于水, 用 $\left(\mathrm{NH}_{1}\right)_{2} \mathrm{SO}$, 为好. 用同一种盐, 吐温 80 浓度愈低, 分相所需盐浓度愈高。 另外, 一完浓度的吐温 80 水溶液形成液一固两相所需盐浓度还与溶液的酸度有关. 溶液酸度 高, 所需盐浓度高. $\mathrm{pH}>2.0$ 后, 分相所需盐浓度恒定. 这可能是在高酸度时, $\mathrm{SO}_{4}^{2-}$ 的质子 化,使 $\left(\mathrm{NH}_{4}\right)_{2} \mathrm{SO}$. 的盐析作用减弱的缘故. 


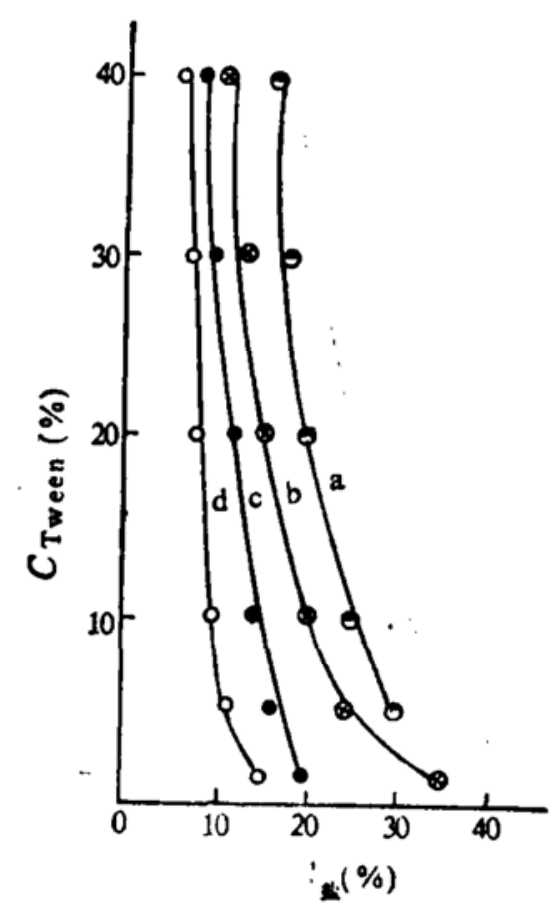

图 1 吐温 80 水溶液分相所需盐浓荌

a. $\mathrm{NaH}_{2} \mathrm{PO}_{4}$; b. $\mathrm{Na}_{3} \mathrm{C}_{6} \mathrm{H}, \mathrm{O}$;

c. $\left(\mathrm{NH}_{4}\right)_{2} \mathrm{SO}_{4} ;$ d. $\mathrm{K}_{2} \mathrm{CO}_{3}$

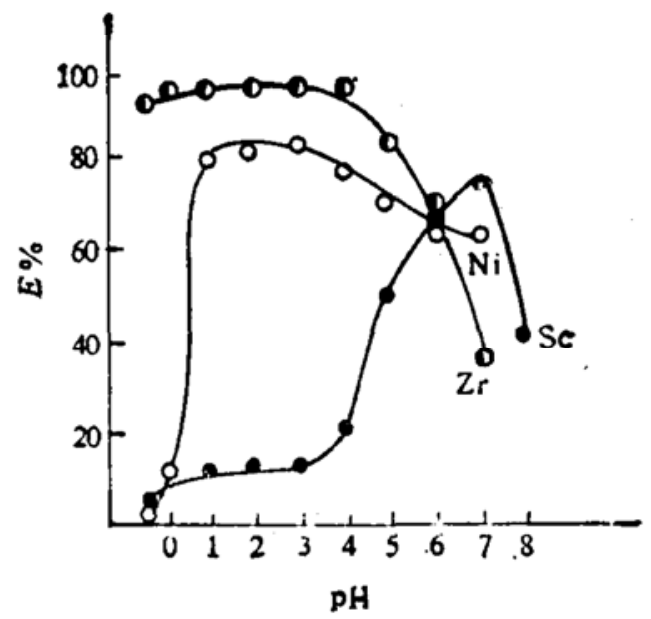

图 $2 \mathrm{pH}-E \%$ 关系曲 $\mathrm{Zr}, \mathrm{Sc} 、 \mathrm{Ni}$ 均为 $50 \mu \mathrm{g}$

2.萃取剂偶续胂 III 在吐温 80- $\left(\mathrm{NH}_{4}\right)_{2} \mathrm{SO}$-水体系中两相间的分配在所研究的 $\mathrm{pH} 0.0-7.0$ 的酸度范围内, 偶氮胂 III 在吐温 80 相中的萃取率均大于 $85 \%$.

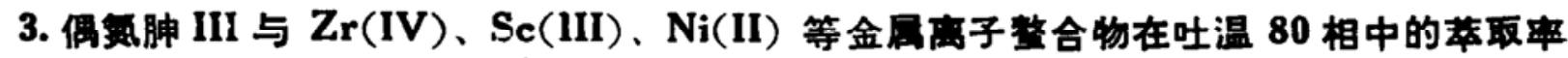

图 2 表明了溶液酸度对 $\mathrm{Zr}(\mathrm{IV}) 、 \mathrm{Sc}(\mathrm{III}) 、 \mathrm{Ni}$ (II) 萃取率的影响。在高酸度时, $\mathrm{Zr}$ (IV) 有 较高的萃取率, 达 $90 \%$ 以上. 而 $\mathrm{Sc}(\mathrm{III}) 、 \mathrm{Ni}(\mathrm{II})$ 的萃取率却较低, 在 $6.0 \mathrm{~mol} \cdot \mathrm{L}^{-1} \mathrm{HCl}$ 介质 中, $\mathrm{Sc}(\mathrm{III}) 、 \mathrm{Ni}(\mathrm{II})$ 的萃取率等于零, 可实现 $\mathrm{Zr}(\mathrm{IV})$ 与 $\mathrm{Sc}(\mathrm{III})$ 或 $\mathrm{Ni}(\mathrm{II})$ 的萃取分离. 另 外, 在 $\mathrm{pH} 1: 0-3.0$ 时, $\mathrm{Sc}$ (III) 有 $80 \%$ 以上的萃取率, $\mathrm{Ni}$ (II) 的萃取率很低, 加人适当的掩 蔽剂,可实现 $\mathrm{Sc}(\mathrm{III})-\mathrm{Ni}(\mathrm{II})$ 的萃取分离.

在适宜的酸度条件下, 萃取剂偶氮胂 III 用量对 $\mathrm{Zr}(\mathrm{IV}) 、 \mathrm{Ni}$ (II) 萃取率的影响如图 3 所 示. 随着萃取剂用量增大, 金属离子趋于鳌合完全, 其萃取率随之增大, 到一定值后, 偶舞胂 III 过量,金属离子会形成不同型体的螯合物 ${ }^{[9]}$, 使其萃取率降低. 值得注意的是,当体系中不 加人萃取剂时,金属离子的萃取率均很低,说明吐温 80 相难以萃取简单的金属离子.

图 4 表示了体系中盐浓度对螯合剂及金属离子萃取率的影响. 其原因是: 盐浓度较低 时, 随 $\left(\mathrm{NH}_{4}\right)_{2} \mathrm{SO}$ 、浓度增高, 吐温 80 相析出完全, 因而螯合物及鳌合剂的萃取率随之增大. 盐 浓度达到一定值, 吐温 80 相已析出完全, 过量盐的强烈盐效应, 使 $\operatorname{Zr}(I V)$ 瞥合物的稳定性 降低, 其萃取率随盐浓度进一步升高而降低, 但盐效应对螯合剂不会有明显影响, 故其萃取率 不随盐浓度而变化。

4. 萃取分离回收试验结果 由图 3 可知, 在 $6.0 \mathrm{~mol} \cdot \mathrm{L}^{-1} \mathrm{HCl}$ 介质中, $\mathrm{Ni}(\mathrm{II}) 、 \mathrm{Sc}(\mathrm{III})$ 不被吐温 80 相萃取而留在水相, $\mathrm{Zr}$ (IV) 的萃取率很高. 在此条件下, 将 $\mathrm{Zr}$ (IV) 萃取两次 就可以达到定量分离 $\mathrm{Zr}$ (IV)-Ni(II) 或 $\mathrm{Zr}$ (IV)-Sc(III) 的目的. 表 1、表 2 是利用该体系 
萃取分离 $\mathrm{Zr}(\mathrm{IV}) 、 \mathrm{Ni}(\mathrm{II})$ 及 $\mathrm{Zr}(\mathrm{IV}) 、 \mathrm{Sc}(\mathrm{III})$ 混合液的回收试验结果。

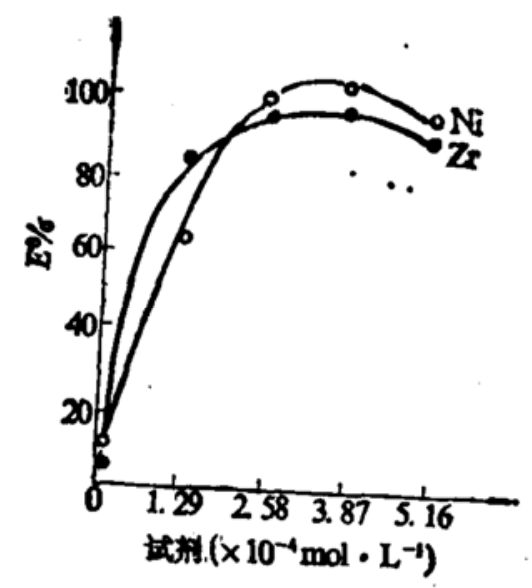

图 3 试剂用盘与 $\mathrm{E} \%$ 关系曲线 $\mathrm{Zr}$ : $\mathrm{pH} 2.0 ; \mathrm{Ni}$ : $\mathrm{pH} 7.0$

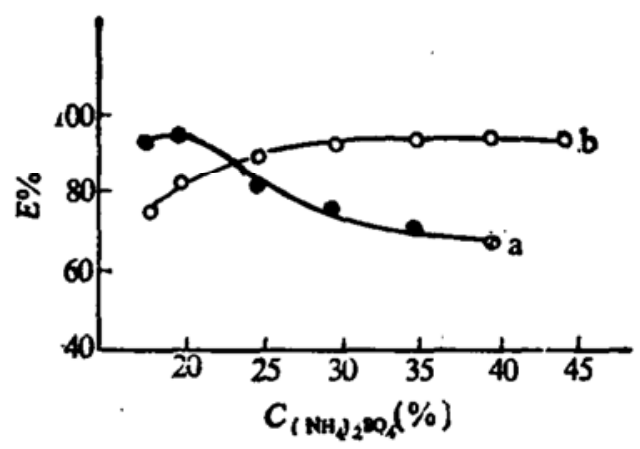

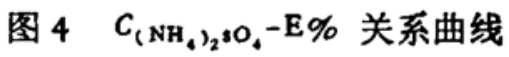

a. $\mathrm{Zr}$ (IV)-偶具胂 III 整合物;

b. 偶筷胂 III

表 $1 \quad \mathrm{Zr}$ (IV)-Ni(II) 定量公取分离回收试验结果

\begin{tabular}{|c|c|c|c|c|c|c|}
\hline 编 号 & $\underset{\left(\mu_{\mathbf{g}}\right)}{\mathrm{Z}_{\mathrm{r}} \text { 加入 }}$ & $\begin{array}{c}\mathrm{Zr} \text { 回收量 } \\
(\mu \mathrm{g})\end{array}$ & $\begin{array}{c}\mathrm{Zr} \text { 回收率 } \\
(\%)\end{array}$ & $\underset{(\mu \mathrm{g})}{\mathrm{Ni} \text { 加入量 }}$ & 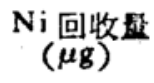 & $\begin{array}{c}\mathrm{Ni} \text { 回收率 } \\
\text { (\%) }\end{array}$ \\
\hline 1 & 25.0 & 25.0 & 100.0 & 25.0 & 24.8 & 99.2 \\
\hline 2 & 50.0 & 52.8 & 105.6 & 10.0 & 9.9 & 99.0 \\
\hline 3 & 10.0 & 10.0 & 100.0 & 50.0 & 48.8 & 97.6 \\
\hline 4 & 5.0 & 5.2 & 104.0 & 100.0 & 96.0 & 96.0 \\
\hline ; & 3.0 & 5.0 & 100.0 & 500.0 & 510.7 & 102.1 \\
\hline 6 & 5.0 & 5.2 & 104.0 & 1000 & 978.7 & 97.9 \\
\hline
\end{tabular}

表 $2 \mathrm{Zr}$ (IV)-Sc(III) 定量萃取分离回收试䠯结果

\begin{tabular}{|c|c|c|c|c|c|c|}
\hline 编号 & $\underset{(\mu \mathrm{r})}{\mathrm{Z} \text { 加人摄 }}$ & $\mathrm{Z}_{\mathrm{r}} \underset{(\mu \mathrm{g})}{(\boldsymbol{\mu})}$ & $\begin{array}{c}\mathrm{Zr} \text { 回收率 } \\
(\%)\end{array}$ & Sc $\underset{(\mu \mathrm{g})}{\text { 加人量 }}$ & $\begin{array}{l}S c \text { 回收量 } \\
\left(\mu_{\mathrm{g}}\right)\end{array}$ & $\begin{array}{c}S c \text { 回收事 } \\
(\%)\end{array}$ \\
\hline 1 & 25.0 & 25.2 & 100.8 & 25.0 & 24.6 & 98.4 \\
\hline 2 & 10.0 & 10.2 & 102.0 & 50.0 & 50.0 & 100.0 \\
\hline 3 & 50.0 & 49.3 & 98.6 & 10.0 & 10.2 & 102.0 \\
\hline 4 & 5.0 & 5.0 & 100.0 & 100.0 & 96.8 & 96.8 . \\
\hline 5 & 5.0 & 5.2 & 104.0 & 500.0 & 486.4 & 97.3 \\
\hline 6 & 5.0 & 5.5 & 110.0 & 1000 & 910.4 & 91.0 \\
\hline
\end{tabular}

\section{考文啫}

[1] Zvarova, T. I. et al., Mikrochimica Acta, III(1984),449-458.

[2]本步海、孙小梅、王孝芳,中南民族学院学报(自然科学版), 1(1987),82-87.

[3] 李步海、孙小梅、杨胜才,高等学校化学学报, 10(1989),3: 293-295.

[4]杭州大学化学系分析化学教研室,分析化学手册(第三分册), 化学工业出版社,北京, 1983,475-477.

‘5]程广䘵、上野贯平、今村君明,有机分析试刘手册,地质出版社, 北京, 1985,128. 